\title{
Multicomponent Phase Behavior Predictions using QSPR-Generalized NRTL and UNIQUAC Models
}

Younas Dadmohammad

Solomon Gebreyohannes

Brian J. Neely

Oklahoma State University

School of Chemical Engineering

Stillwater, OK 74078-0537

Khaled A. M. Gasem*

University of Wyoming

Department of Chemical \& Petroleum Engineering

Laramie, WY 82071

* email: gasem@uwyo.edu

Phone: 405-766-2845 


\begin{abstract}
Multiphase, multicomponent equilibrium systems are encountered commonly in the chemical industry. The non-random two-liquid (NRTL) and universal quasi-chemical (UNIQUAC) models are applied widely to correlate activity coefficients in phase equilibria calculations. We demonstrated previously the predictive capability of a quantitative structure-property relationship (QSPR)-generalized NRTL model (NRTL-QSPR) for vapor-liquid equilibrium (VLE) property predictions of diverse binary systems. In this study, we further evaluate the prediction quality of the NRTL and UNIQUAC models as they apply to multicomponent systems. Specifically, we evaluate both the representation and prediction of vapor-liquid equilibrium properties of multicomponent systems using regressed and QSPR-generalized parameters of the constituent binaries.

To complete this evaluation, a database comprised of 57 ternary VLE systems and their 75 constituent binary systems was assembled. In addition, 29 ternary systems were employed to examine the efficacy of the models when at least one constituent binary is missing.

For ternary systems where all three constituent binary systems were available, the NRTL-QSPR model provided overall percent absolute average deviations (\%AADs) of 3.7, 0.3, 9.0, and 8.7 for pressure $(P)$, temperature $(T)$, mole fraction $\left(y_{1}\right)$, and $K$-values, respectively. In comparison, the generalized UNIQUAC-QSPR model exhibited errors of 3.5, 0.3, 8.2, and 7.9, respectively. Thus, for the systems considered, both models exhibit comparable accuracy. Further, for these systems the group-contribution method Modified UNIFAC (mUNIFAC) produced slightly better results.

For systems where at least one constituent binary is missing in our database, errors for the NRTL-QSPR are 6.3, 0.5, 11.2 and 11.4\%AADs for $P, T, y_{1}$ and $K$-values, respectively. The comparable errors for the UNIQUAC-QSPR for this case are 8.4, 0.7, 12.9 and 12.5, respectively. For the same set of ternary systems, the mUNIFAC model produced \%AADs of 20.7, 2.1, 25.7 and 26.7 for $P, T, y_{1}$ and $K$-values, respectively. As such, the mUNIFAC model results in three times higher errors than our generalized models. Although additional evaluation using a comprehensive database is required, the results presented in this study demonstrate the efficacy of the QSPR binary parameter generalizations, as applied to the NRTL and UNIQAC models, for VLE multicomponent property predictions.
\end{abstract}

Keywords: Ternary Systems, Phase Behavior Prediction, Parameter Generalization, NRTL, UNIQUAC and QSPR 


\section{Introduction}

Development of multiphase, multicomponent separation processes can be affected adversely by unresolved complexity in the phase behavior description [1]. As such, several experimental approaches have been developed for measuring the equilibrium properties of multiphase, multicomponent systems; however, these techniques are challenging, time consuming, costly, and often mixture specific. Further, the number of chemicals for which measured data are available is at most several thousands, while the number of compounds of interest to the chemical industry, is in excess of 100,000 and rapidly growing [2].

Thus, predictive models have been developed to reduce the experimental burden. Currently, several models are used routinely for this purpose, including mUNIFAC, ASOG [3, 4], and models based on quantum chemical calculations such as the conductor-like screening model for real solvents (COSMO-RS) [5-7] which are used for a priori prediction purposes.

Although these models provide useful predictions for many applications, their predictive reliability continues to be the focus of researchers and practitioners alike. For example, the potential benefits of a group-contribution model such as mUNIFAC can be affected by limitations such as the inability to define effectively the functional groups of some chemical species and a lack of model interaction parameters for functional groups that are not represented in the data matrix. In contrast, the COSMO-RS model is more universal compared to the mUNIFAC model since COSMO-RS relies on individual chemical elements as opposed to functional groups. For some polar systems, however, the COSMO-RS model results in worse predictions than the mUNIFAC model [8] and moreover, sometimes fails to describe the VLE of even nearly-ideal organic systems [8, 9].

Hence, a need still exists for reliable and accurate thermodynamic models capable of providing a priori predictions of the multiphase, multicomponent behavior of diverse systems in the absence of experimental data.

In the context of this study, our developed models are deemed viable for a priori multiphase, multicomponent property predictions if they meet the following conditions pertaining to internal and external model consistency:

1. Ternary and multicomponent systems can be represented precisely using regressed parameters obtained from binary systems.

2. Ternary and multicomponent systems can be predicted accurately using structure-based generalized binary parameters. 
Substantial progress has been made in the representation of multicomponent vapor-liquid equilibria [see, e.g., [10-13]]. The NRTL, UNIQUAC, and Wilson models, for example, demonstrate well the current capability of representing multicomponent phase behavior relying on only binary (two-body interactions) parameters provided by binary mixtures. As such, the focus of this study is on generalized binary interaction parameters for multicomponent predictions.

In previous work [14], we have demonstrated the predictive capability of a quantitative structureproperty relationship generalized NRTL model (NRTL-QSPR) for vapor-liquid equilibrium (VLE) property predictions binary systems. In this study, we further evaluate the prediction quality of the NRTL and UNIQUAC models as they apply to multicomponent systems. Specifically, we assess both the representation and prediction of vapor-liquid equilibrium properties of multicomponent systems using regressed and QSPR-generalized parameters of the constituent binaries.

Our basic premise is that using the theory-framed QSPR modeling approach, the NRTL and UNIQUAC interaction parameters generalizations would be capable of providing binary and multicomponent VLE system phase behavior predictions within twice the error of model representations based on parameters regressed from binary experimental data.

For this purpose, a database of VLE experimental measurements involving 57 ternary systems was used to conduct evaluations. This database includes more than 2500 data points for 75 unique binary systems. Further, the predictive capability of generalized models are compared with those of a group-contribution approach as exemplified by mUNIFAC [15]. It should be noted that the latest mUNIFAC group interaction parameters are available online through the company consortium.

\section{Overview of activity coefficient models}

Both the NRTL and UNIQUAC excess Gibbs models are well suited for correlating multiphase, multicomponent equilibrium data for a wide range of conditions [1]. The following sections provide an applicative overview of the two models.

\subsection{Non-Random Two-Liquid (NRTL) model}

Renon and Prausnitz [12] integrated the concepts of local composition, advanced by Wilson [16], and the two-liquid solution theory of Scott to develop the NRTL excess Gibbs energy model. For a binary system, the NRTL is written as follows: 
$\frac{g^{E}}{R T}=x_{1} x_{2}\left(\frac{\tau_{21} G_{21}}{x_{1}+x_{2} G_{21}}+\frac{\tau_{12} G_{12}}{x_{2}+x_{1} G_{12}}\right)$

The resulting expressions for the binary activity coefficients, $\gamma$, are

$\ln \gamma_{1}=x_{2}^{2}\left[\tau_{21}\left(\frac{G_{21}}{x_{1}+x_{2} G_{21}}\right)^{2}+\frac{\tau_{12} G_{12}}{\left(x_{2}+x_{1} G_{12}\right)^{2}}\right]$

and

$\ln \gamma_{2}=x_{1}^{2}\left[\tau_{12}\left(\frac{G_{12}}{x_{2}+x_{1} G_{12}}\right)^{2}+\frac{\tau_{21} G_{21}}{\left(x_{1}+x_{2} G_{21}\right)^{2}}\right]$

where the model parameters $\tau_{12}$ and $G_{12}$ are defined as

$G_{12}=\exp \left(-\alpha_{12} \tau_{12}\right) \quad \& \quad G_{21}=\exp \left(-\alpha_{12} \tau_{21}\right)$

and

$\tau_{12}=\frac{g_{12}-g_{22}}{R T}=\frac{a_{12}}{T} \quad \tau_{21}=\frac{g_{21}-g_{11}}{R T}=\frac{a_{21}}{T}$

Here, $g_{12}$ is an energy parameter that characterizes interactions between molecules 1 and 2 , $\alpha_{12}$ is the non-randomness factor in the mixture, $x_{i}$ is the mole fraction of component $i, R$ is the universal gas constant, and $T$ is the mixture temperature.

The NRTL equation contains three parameters that can be specified for a binary system. These adjustable parameters are $g_{12}-g_{22}, g_{21}-g_{11}$, and $\alpha_{12}$. To be consistent with the DECHEMA database [17] in accommodating liquid-liquid equilibrium systems, the non-randomness factor ( $\alpha_{12}$ ) was kept constant as 0.2 for all binary systems. The validity of this assumption was further confirmed in our recent study [14].

The NRTL model, as shown in Equation 1, can be applied for a solution of $m$ components, as follows:

$\frac{g^{E}}{R T}=\sum_{i=1}^{m} x_{i} \frac{\sum_{j=1}^{m} \tau_{j i} G_{j i} x_{j}}{\sum_{l=1}^{m} G_{l i} x_{l}}$

where

$\tau_{j i}=\frac{g_{j i}-g_{i i}}{R T}$

and

$G_{j i}=\exp \left(-\alpha_{j i} \tau_{j i}\right) \quad \alpha_{j i}=\alpha_{i j}$ 
The activity coefficient for any component $i$ is then given by:

$\ln \gamma_{i}=\frac{\sum_{j=1}^{m} \tau_{j i} G_{j i} x_{j}}{\sum_{l=1}^{m} G_{l i} x_{l}}+\sum_{j=1}^{m} \frac{x_{j} G_{i j}}{\sum_{l=1}^{m} G_{l j} x_{l}}\left(\tau_{i j}-\frac{\sum_{r=1}^{m} \tau_{r j} G_{r j} x_{r}}{\sum_{l=1}^{m} G_{l j} x_{l}}\right)$

As stated earlier, while Equations 6-9 apply to multicomponent systems, the model requires only binary parameters.

\subsection{Universal Quasi-Chemical (UNIQUAC) model}

The UNIQUAC model developed by Abrams and Prausnitz in 1975 [18]. Herein, the excess Gibbs energy is expected as the sum of the size and shape dependent combinatorial part to describe the dominant entropic contribution, and the energy interaction dependent residual part to account for intermolecular forces. The development of the UNIQUAC model resulted from a critical examination of the NRTL model, which revealed that this activity coefficient model is more suitable for excess properties such as $h^{E}$ rather than $g^{E}$ [19].

This model was derived from a statistical-mechanical basis extending Guggenheim's quasichemical theory [18]. All parameters for a ternary system can be obtained from binary VLE data using the three binary combinations of the ternary components. The UNIQUAC equation is expressed, as follows:

$\frac{g^{E}}{R T}=\left(\frac{g^{E}}{R T}\right)_{\text {Combinatorial }}+\left(\frac{g^{E}}{R T}\right)_{\text {Residual }}$

for a binary mixture,

$\left(\frac{g^{E}}{R T}\right)_{\text {Combinatorial }}=x_{1} \ln \frac{\Phi_{1}^{*}}{x_{1}}+x_{2} \ln \frac{\Phi_{2}^{*}}{x_{2}}+\frac{z}{2}\left(x_{1} q_{1} \ln \frac{\theta_{1}}{\Phi_{1}^{*}}+x_{2} q_{2} \ln \frac{\theta_{2}}{\Phi_{2}^{*}}\right)$

and

$\left(\frac{g^{E}}{R T}\right)_{\text {Residual }}=-x_{1} q_{1}^{\prime} \ln \left(\theta_{1}^{\prime}+\theta_{2}^{\prime} \tau_{21}\right)-x_{2} q_{2}^{\prime} \ln \left(\theta_{2}^{\prime}+\theta_{1}^{\prime} \tau_{12}\right)$

where the coordination number $z$ is set equal to 10. $q$ and $q^{\prime}$ are pure component moleculestructure constants depending on molecular size and external surface areas. In the original formulation, $q=q^{\prime}$. The two adjustable parameters, $\tau_{12}$ and $\tau_{21}$, are calculated in terms of characteristic energies. The volume fraction, $\Phi^{*}$, and surface area fractions, $\theta$ and $\theta^{\prime}$, for each component are given as:

$\Phi_{1}^{*}=\frac{x_{1} r_{1}}{x_{1} r_{1}+x_{2} r_{2}} \quad \Phi_{2}^{*}=\frac{x_{2} r_{2}}{x_{1} r_{1}+x_{2} r_{2}}$ 
and

$\theta_{1}=\frac{x_{1} q_{1}}{x_{1} q_{1}+x_{2} q_{2}} \quad \theta_{2}=\frac{x_{2} q_{2}}{x_{1} q_{1}+x_{2} q_{2}}$

and

$\theta_{1}^{\prime}=\frac{x_{1} q_{1}^{\prime}}{x_{1} q_{1}^{\prime}+x_{2} q_{2}^{\prime}} \quad \theta_{2}^{\prime}=\frac{x_{2} q_{2}^{\prime}}{x_{1} q_{1}^{\prime}+x_{2} q_{2}^{\prime}}$

where the parameter $r$ is a pure component molecule-structure constant depending on molecular size and external surface area. The resultant expressions for the activity coefficients of a binary mixture are:

$\ln \gamma_{1}=\ln \frac{\Phi_{1}^{*}}{x_{1}}+\frac{z}{2} q_{1} \ln \frac{\theta_{1}}{\Phi_{1}^{*}}+\Phi_{2}^{*}\left(l_{1}-\frac{r_{1}}{r_{2}} l_{2}\right)-q_{1}^{\prime} \ln \left(\theta_{1}^{\prime}+\theta_{2}^{\prime} \tau_{21}\right)+\theta_{2}^{\prime} q_{1}^{\prime}\left(\frac{\tau_{21}}{\theta_{1}^{\prime}+\theta_{2}^{\prime} \tau_{21}}-\frac{\tau_{12}}{\theta_{2}^{\prime}+\theta_{1}^{\prime} \tau_{12}}\right)$

and

$\ln \gamma_{2}=\ln \frac{\Phi_{2}^{*}}{x_{2}}+\frac{z}{2} q_{2} \ln \frac{\theta_{2}}{\Phi_{2}^{*}}+\Phi_{2}^{*}\left(l_{2}-\frac{r_{2}}{r_{1}} l_{1}\right)-q_{2}^{\prime} \ln \left(\theta_{2}^{\prime}+\theta_{1}^{\prime} \tau_{12}\right)+\theta_{1}^{\prime} q_{2}^{\prime}\left(\frac{\tau_{12}}{\theta_{2}^{\prime}+\theta_{1}^{\prime} \tau_{12}}-\frac{\tau_{21}}{\theta_{1}^{\prime}+\theta_{2}^{\prime} \tau_{21}}\right)$

where

$l_{1}=\frac{z}{2}\left(r_{1}-q_{1}\right)-\left(r_{1}-1\right) \quad l_{2}=\frac{z}{2}\left(r_{2}-q_{2}\right)-\left(r_{2}-1\right)$

Equations 10-12 give the excess Gibbs energy for a binary mixture in terms of two adjustable binary parameters and two pure-component structural parameters, $r$ and $q$. The derivation of Equations 16 and 17 can be extended readily to mixtures containing three or more components without additional assumptions [18]. For multicomponent systems, the activity coefficient for a specific component, $i$, is:

$\ln \gamma_{i}=\ln \frac{\Phi_{i}^{*}}{x_{i}}+\frac{z}{2} q_{i} \ln \frac{\theta_{i}}{\Phi_{i}^{*}}+l_{i}-\frac{\Phi_{i}^{*}}{x_{i}} \sum_{j=1}^{m} x_{j} l_{j}-q_{i}^{\prime} \ln \left(\sum_{j=1}^{m} \theta_{j}^{\prime} \tau_{j i}\right)+q_{i}^{\prime}-q_{i}^{\prime} \sum_{j=1}^{m} \frac{\theta_{j}^{\prime} \tau_{i j}}{\sum_{k=1}^{m} \theta_{k}^{\prime} \tau_{k j}}$

This equation requires only pure-component and binary parameters. The values of the van der Waals surface area $\left(q_{i}\right)$ and volume $\left(r_{i}\right)$ in all above equations are obtained from experimental data, as found in DECHEMA [17], and then the interaction parameters are regressed using these data.

\subsection{Generalized NRTL and UNIQUAC models, QSPR}

Although the NRTL and UNIQUAC models, as described above, are capable for describing phase equilibrium properties, they require relevant experimental data on the binary systems 
considered. Therefore, a need exists for generalized interaction parameters to facilitate predictions when such binary data are lacking.

In our previous study, a method for generalizing the binary interaction parameters of the NRTL and UNIQUAC models was developed [14]. A theory-framed quantitative structure-property relationship (QSPR) modeling approach was applied to generalize these binary interaction parameters. The NRTL-QSPR and UNIQUAC-QSPR model parameter generalizations produced accurate predictions for the diverse binary systems considered.

In this study, the correlative and predictive abilities of the NRTL and UNIQUAC models, as they apply to multicomponent system, are examined. Specifically, we evaluate the representation and prediction of vapor-liquid equilibrium properties of multicomponent systems using regressed and QSPR-generalized parameters of the constituent binaries. Further, the predictive capability of generalized models is compared with those of the group-contribution approach, as exemplified by mUNIFAC.

\section{Methodology}

Our NRTL-QSPR and UNIQUAC-QSPR binary parameters were constructed using binary systems [14]. In the current work, the regressed (internal consistency testing) and predicted (external consistency testing) parameters for the NRTL and UNIQUAC models are applied to multiphase, multicomponent systems. Two model evaluation scenarios are considered. First, we study 57 ternary systems, where both models employ the required constituent binaries in the database. This signifies the use of the full information set, as represented by the binary interaction parameters. Second, we use 29 ternary systems to investigate the quality of generalized predictions when at least one constituent binary was not part of the list of binaries used to develop the QSPR models. Here, we examine the inherent ability of the model when binary-specific interaction information is lacking.

\subsection{Database}

A representative database of reliable data is the prime requirement for developing a model and evaluating its performance [9]. The database used in this research was compiled from the following three sources: (1) a low-pressure binary VLE database, Oklahoma State University, OSU database [20], (2) DECHEMA VLE compilation [17], and (3) DIPPR [21]. The VLE data were integrated into one database, which is comprised of 75 binary systems with more than 2500 data points. We have studied 86 ternary systems created using the binary data set. The selected systems for the VLE database were chosen on the basis of consistency test 
information provided in the DECHEMA Chemistry Data Series [17]. The data covered a temperature range from 128 to $554 \mathrm{~K}$ and pressures to 58 bar; however, over $99 \%$ of the data were at a pressure of less than 10 bar. The 35,000 assembled experimental data points are a combination of both isothermal and isobaric data. The required pure-component vapor pressure data were collected from DIPPR [17] and DECHEMA [12].

\subsection{NRTL and UNIQUAC parameters regression methodology}

Optimum binary interaction parameters for the NRTL $\left(a_{12}, a_{21}\right)$ and UNIQUAC $(r, q)$ models were obtained through regression analysis. Specifically, the following equilibrium closed-system criteria were applied during regression for the coexisting vapor and liquid phases, subject to mass balance constraints:

$P^{v}=P^{l}$

$T^{v}=T^{l}$

$\hat{f}_{i}^{v}=\hat{f}_{i}^{l} \quad i=1, \ldots, N$

where $\hat{f}_{i}$ is the partial fugacity of component $i$ in the mixture, $T$ is the temperature, $P$ is the pressure, and the superscripts, $v$ and $l$, indicate vapor and liquid, respectively. In the regression analyses, the conventional split approach was used for VLE system modeling:

$\widehat{\emptyset}_{i}^{v} P y_{i}=\gamma_{i} P_{i}^{o} \emptyset_{i}^{v} x_{i} \lambda_{i}$

where for component $i, \widehat{\emptyset}_{i}^{v}$ is the component partial fugacity coefficient in the vapor phase, $y_{i}$ is the vapor mole fraction, $\gamma_{i}$ is the component activity coefficient in the liquid phase, $P_{i}^{0}$ is the pure-component vapor pressure, $\emptyset_{i}^{v}$ is the pure-component fugacity coefficient in the vapor phase, $x_{i}$ is the liquid mole fraction, and $\lambda_{i}$ is the Poynting factor. In this work, vapor-phase fugacity coefficients were assumed to be one since the VLE systems employed are at low pressure.

Another crucial aspect of regression analysis is the choice of a suitable objective function, $O F$. In this work, the $O F$ is expressed for the two models and for binary systems by the sum of squares of relative errors in pressure and the activity coefficients of the two components:

$\min O F=\sum_{i=1}^{n}\left(\frac{P^{E x p}-P^{C a l c}}{P^{E x p}}\right)_{i}^{2}+\sum_{i=1}^{n}\left(\frac{\gamma_{1}^{E x p}-\gamma_{1}^{\text {Calc }}}{\gamma_{1}^{E x p}}\right)_{i}^{2}+\sum_{i=1}^{n}\left(\frac{\gamma_{2}^{E x p}-\gamma_{2}^{\text {Calc }}}{\gamma_{2}^{E x p}}\right)_{i}^{2}$ 
where $n$ is the number of data points, the superscripts Exp and Calc refer to experimental and calculated values, respectively, and the subscripts 1 and 2 refer to the binary components [14]. This objective function was developed after evaluating the VLE property representations employing various objective function formulations. The detailed study on parameter regressions based on the NRTL and UNIQUAC models were presented in our previous study [14].

In addition to pressure and activity coefficient prediction assessment, other equilibrium properties such as temperature and component equilibrium $K$-values of each binary system are also investigated. The equilibrium $K$-value for component $i$ is the ratio of the vapor to liquid mole fraction, which can be modified as follows:

$K_{i}=\frac{y_{i}}{x_{i}}=\frac{\gamma_{i} P_{i}^{o} \phi_{i}^{v} x_{i} \lambda_{i}}{\widehat{\emptyset}_{i}^{v} P y_{i}}$

\subsection{Case studies}

To achieve the objectives of this study and facilitate evaluation of the predictive capability of the NRTL and UNIQUAC models, seven case studies were constructed. Since low-pressure VLE mixtures are used in this work, the ideal gas (IG) model was proposed to describe the gas phase behavior for all case studies. Following is a description of the case studies employed:

Case I, Ideal Solution: This case illustrates the quality of phase behavior predictions assuming ideal conditions and relying solely on pure-fluid properties. This case provides the phase behavior predictions of the reference model absent all information from binary data.

Case II, NRTL-Regressed: The NRTL model parameters are regressed directly from binary VLE experimental data, and then applied for ternary mixture representations. This case provides the benchmark for the precision of the NRTL model for ternary systems, using only binary data (two-body interaction assumption).

Case III, NRTL-QSPR: The binary interaction parameters are predicted using the QSPR generalized model (NRTL-QSPR). Here, the parameters are predicted for the constituent 75 binary systems of the 57 ternary systems studied. This case assesses the predictive abilities of generalized model.

Case IV, NRTL-QSPR/MCB ${ }^{1}$ : As in the case above, the binary interaction parameters are predicted using the QSPR generalized model (NRTL-QSPR). In this case, however, we

\footnotetext{
${ }^{1}$ Missing at least one constituent binary (MCB)
} 
evaluate the predictive abilities of the generalized model for 29 ternary systems where at least one of the constituent binaries was missing from the database used for model development.

Cases V, VI, and VII: These cases apply to the UNIQUAC model evaluation. They are comparable to Cases II, III, and IV in methodology. As such, the UNIQUAC-Regressed, UNIQUAC-QSPR, and UNIQUAC-QSPR/MCB models are used in Cases V, VI, and VII, respectively.

All regressions were completed by performing bubble-point pressure calculations. For all case studies, regressed or QSPR predicted parameters are used directly to calculate (a) $P, y_{1}$ and $K$ values for known $T$ and $x_{1}$, and (b) $T$ for known $P$ and $x_{1}$. As expected, using regressed NRTL and UNIQUAC binary interaction parameters (Cases II and V) resulted in the best model predictions; thus, the performance metrics of these cases are employed on a comparative basis to assess the performance of the predictive models.

\section{Results and discussion}

In this section, the results for representation and predictive capability of each model in each case for both binary and ternary systems are presented. To compare with current groupcontributions modeling capabilities in the literature, we applied mUNIFAC [15] to the same set of ternary systems.

\subsection{Ideal solution model}

Table 1 presents the phase behavior property predictions for the Ideal Solution model (Case I) involving 75 unique binary systems and 57 ternary systems. As expected, the model is not accurate when dealing with phase behavior of nonideal systems. Specifically, the application of the Ideal Solution model to the binary systems considered resulted in overall percent absolute average deviations (\%AADs) of 13.9, 1.4, 16.9, and 21.0 for $P, T, y_{1}$, and $K$-values, respectively. As nonideality is not accounted for in this case, the property errors for the ternary systems are much higher than the binary systems with overall errors of $60.8,8.1,55.5$, and 57.7 \%AADs for $P, T, y_{1}$, and $K$-values, respectively. For our evaluations, we have considered 75 binary and 57 ternary mixtures; however, due to a lack of vapor-phase experimental data, there may be fewer systems to represent the properties of interest beyond pressure. 


\subsection{NRTL models}

The results for the NRTL-Regressed and NRTL-QSPR models for both binary and ternary systems are presented in Tables 2-4. Using the NRTL-Regressed (Case II) parameters for 75 unique binary systems resulted in overall \%AADs of $1.7,0.2,3.2$, and 3.9 for $P, T, y_{1}$, and $K$ values, respectively. The extension of binary parameters from the NRTL-Regressed model for the 57 ternary systems shows good results with \%AADs of $3.0,0.3,8.7$, and 8.3 for $P, T, y_{1}$, and $K$-values, respectively. The ternary results show errors within two times the errors produced by the binary systems. The quality of representation here is a reflection of the ultimate abilities of the model under the two-body interaction assumption.

Table 1: Case I results for binary and ternary system phase behavior predictions

\begin{tabular}{|c|c|c|c|c|c|c|c|}
\hline Model & System & Property & No. of sys. & No. of pts. & RMSE & Bias & $\% A A D$ \\
\hline \multirow{4}{*}{$\begin{array}{c}\text { IG / } \\
\text { Ideal Solution }\end{array}$} & \multirow{4}{*}{ Binary } & $\mathrm{P}$ (bar) & \multirow{2}{*}{75} & \multirow{2}{*}{2992} & 0.20 & -0.10 & 13.9 \\
\hline & & $\mathrm{T}(\mathrm{K})$ & & & 7.30 & 4.30 & 1.4 \\
\hline & & $\mathrm{y}_{1}$ & \multirow{2}{*}{63} & \multirow{2}{*}{1818} & 0.10 & -0.02 & 16.9 \\
\hline & & K-values & & & 2.80 & -0.60 & 21.0 \\
\hline \multirow{4}{*}{$\begin{array}{c}\text { IG / } \\
\text { Ideal Solution }\end{array}$} & \multirow{4}{*}{ Ternary } & $\mathrm{P}$ (bar) & \multirow{2}{*}{57} & \multirow{2}{*}{2212} & 43.60 & 4.10 & 60.8 \\
\hline & & $\mathrm{T}(\mathrm{K})$ & & & 0.20 & 26.2 & 8.1 \\
\hline & & $\mathrm{y}_{1}$ & \multirow{2}{*}{52} & \multirow{2}{*}{1890} & 0.20 & 0.20 & 55.5 \\
\hline & & K-values & & & 3.00 & 0.80 & 57.7 \\
\hline
\end{tabular}

Table 2: Case II results for binary and ternary system phase behavior representations

\begin{tabular}{|c|c|c|c|c|c|c|c|}
\hline Model & System & Property & No. of sys. & No. of pts. & RMSE & Bias & $\% A A D$ \\
\hline \multirow{4}{*}{ IG / NRTL } & \multirow{4}{*}{ Binary } & $\mathrm{P}$ (bar) & \multirow{2}{*}{75} & \multirow{2}{*}{2992} & 0.00 & -0.003 & 1.7 \\
\hline & & $\mathrm{T}(\mathrm{K})$ & & & 2.50 & 0.175 & 0.2 \\
\hline & & $\mathrm{y}_{1}$ & \multirow{2}{*}{63} & \multirow{2}{*}{1818} & 0.00 & 0.001 & 3.2 \\
\hline & & K-values & & & 0.80 & -0.044 & 3.9 \\
\hline \multirow{4}{*}{ IG / NRTL } & \multirow{4}{*}{ Ternary } & $\mathrm{P}$ (bar) & \multirow{2}{*}{57} & \multirow{2}{*}{2212} & 0.00 & -0.004 & 3.0 \\
\hline & & $\mathrm{T}(\mathrm{K})$ & & & 1.60 & 0.162 & 0.3 \\
\hline & & $\mathrm{y}_{1}$ & \multirow{2}{*}{52} & \multirow{2}{*}{1890} & 0.00 & 0.002 & 8.7 \\
\hline & & K-values & & & 0.40 & 0.011 & 8.3 \\
\hline
\end{tabular}


The results of the NRTL-QSPR model (Case III) for the binary and ternary systems provide evidence for the predictive abilities of the generalized model. For the binary systems, the overall \%AADs are 3.0, 0.3, 4.7, and 6.0 for $P, T, y_{1}$, and $K$-values, respectively. Similarly, for the ternary systems, \%AADs of 3.7, 0.3, 9.0, and 8.7 are observed for $P, T, y_{1}$, and $K$-values, respectively. In general, we are seeking generalization prediction errors within two times of those produced by data regressions. The current results, as indicated by the ratio metric in Tables 2 and 3, are in support of this intension.

Table 3: Case III results for binary and ternary system phase behavior predictions

\begin{tabular}{|c|c|c|c|c|c|c|c|c|}
\hline Model & System & Property & No. of sys. & No. of pts. & RMSE & Bias & \%AAD & $\begin{array}{c}\text { Gen/Reg } \\
\text { Ratio }\end{array}$ \\
\hline \multirow{4}{*}{$\begin{array}{c}\text { IG / } \\
\text { NRTL- } \\
\text { QSPR }\end{array}$} & \multirow{4}{*}{ Binary } & $\mathrm{P}$ (bar) & \multirow{2}{*}{75} & \multirow{2}{*}{3209} & 0.10 & -0.002 & 3.0 & 1.8 \\
\hline & & $\mathrm{T}(\mathrm{K})$ & & & 2.60 & 0.303 & 0.3 & 1.5 \\
\hline & & $\mathrm{y}_{1}$ & \multirow{2}{*}{63} & \multirow{2}{*}{1817} & 0.00 & -0.002 & 4.7 & 1.5 \\
\hline & & K-values & & & 1.10 & -0.098 & 6.0 & 1.5 \\
\hline \multirow{4}{*}{$\begin{array}{l}\text { IG / } \\
\text { NRTL- } \\
\text { QSPR }\end{array}$} & \multirow{4}{*}{ Ternary } & $\mathrm{P}$ (bar) & \multirow{2}{*}{57} & \multirow{2}{*}{2212} & 0.10 & -0.007 & 3.7 & 1.2 \\
\hline & & $\mathrm{T}(\mathrm{K})$ & & & 1.80 & 0.286 & 0.3 & 1.0 \\
\hline & & $\mathrm{y}_{1}$ & \multirow{2}{*}{52} & \multirow{2}{*}{1890} & 0.00 & 0.002 & 9.0 & 1.0 \\
\hline & & K-values & & & 0.40 & 0.001 & 8.7 & 1.0 \\
\hline
\end{tabular}

Table 4 shows the results for Case IV, which involved 29 ternary systems with at least one missing constituent binary. The NRTL-QSPR model was utilized to predict the parameters employed. For this case, overall \%AADs of 6.3, 0.5, 11.2 and 11.4 are indicated for $P, T, y_{1}$, and $K$-values predictions, respectively. This demonstrates the ability of the NRTL-QSPR model to predict equilibrium phase properties of multicomponent mixtures when some constituent binaries are not represented in the database used for model development. The resulting property prediction errors are less than twice the error observed in comparison to the results from Case III, where all the constituent binary systems were included in the database. 
Table 4: Case IV results for binary and ternary system phase behavior predictions with missing constituent binaries

\begin{tabular}{|c|c|c|c|c|c|c|}
\hline Model & Property & No. of sys. & No. of pts. & RMSE & Bias & $\% A A D$ \\
\hline \multirow{4}{*}{$\begin{array}{c}\text { IG / } \\
\text { NRTL-QSPR }\end{array}$} & $\mathrm{P}$ (bar) & \multirow{2}{*}{29} & \multirow{2}{*}{981} & 0.09 & 0.02 & 6.3 \\
\hline & $\mathrm{T}(\mathrm{K})$ & & & 2.60 & -0.33 & 0.5 \\
\hline & $\mathrm{y}_{1}$ & \multirow{2}{*}{29} & \multirow{2}{*}{966} & 0.06 & 0.01 & 11.2 \\
\hline & K-values & & & 1.71 & -0.04 & 11.4 \\
\hline
\end{tabular}

Figures 1-4 show the distribution of the overall errors for the prediction of pressure and temperature of binary and ternary systems using the NRTL-QSPR model parameters for Case III. The errors produced by the NRTL-QSPR model for pressure and temperature predictions for the binary and ternary systems are, respectively, less than 3.0 and 0.4 for over $50 \%$ of the data considered. 


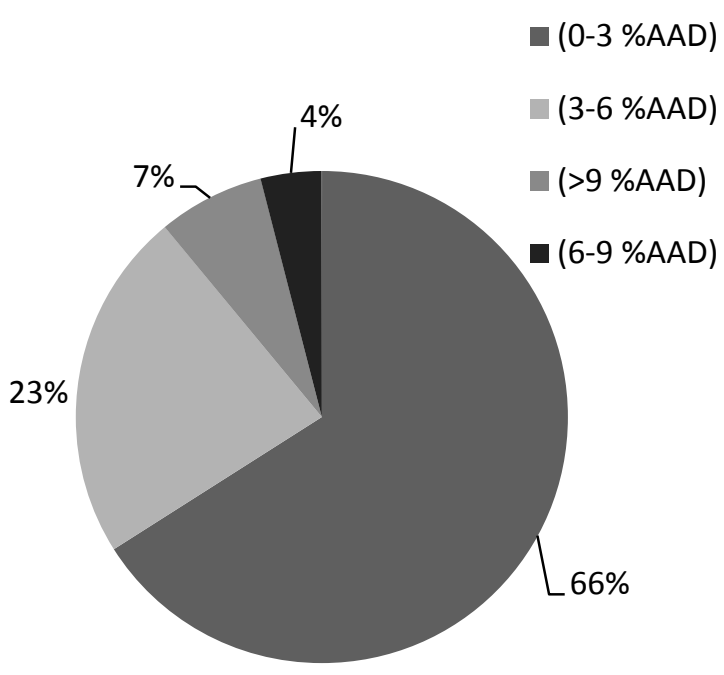

Figure 1: The error distribution of pressure predictions for binary systems using the NRTL-QSPR

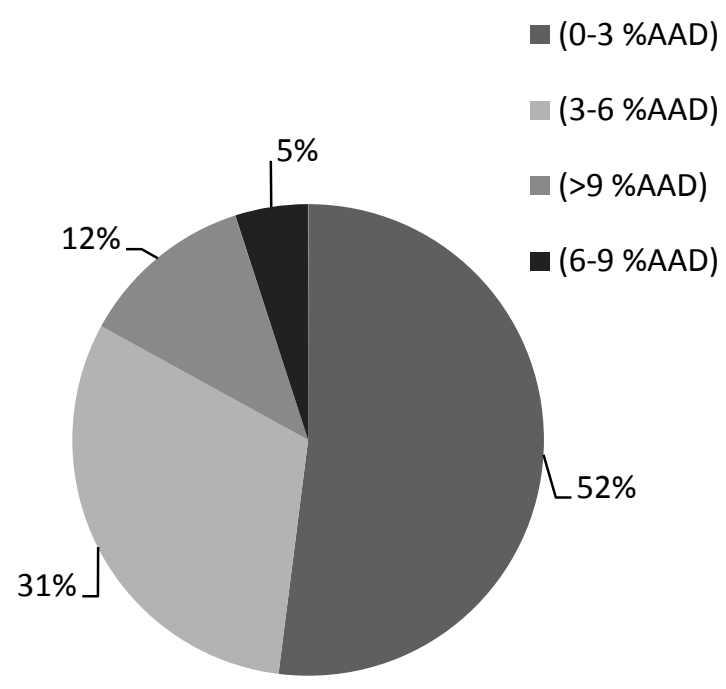

Figure 3: The error distribution of pressure predictions for ternary systems using the NRTL-QSPR

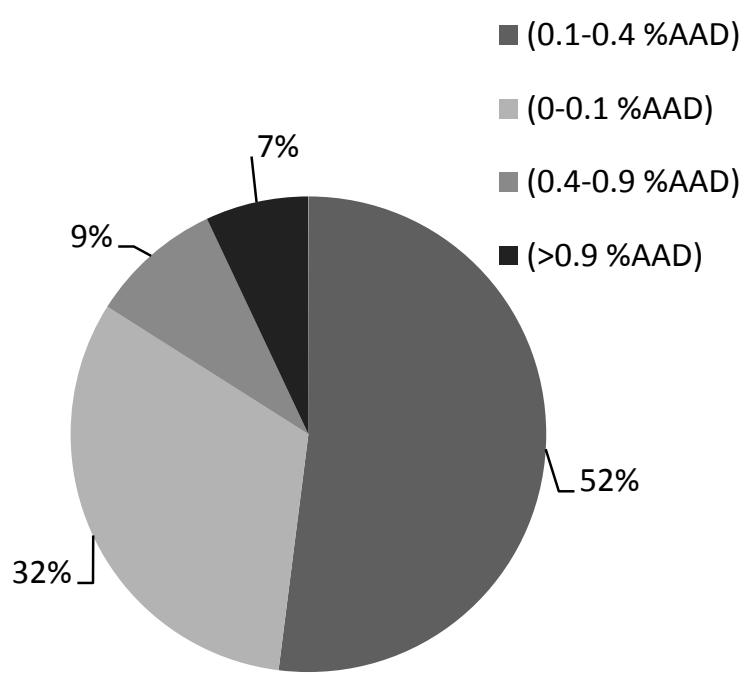

Figure 2: The error distribution of temperature predictions for binary systems using the NRTL-QSPR

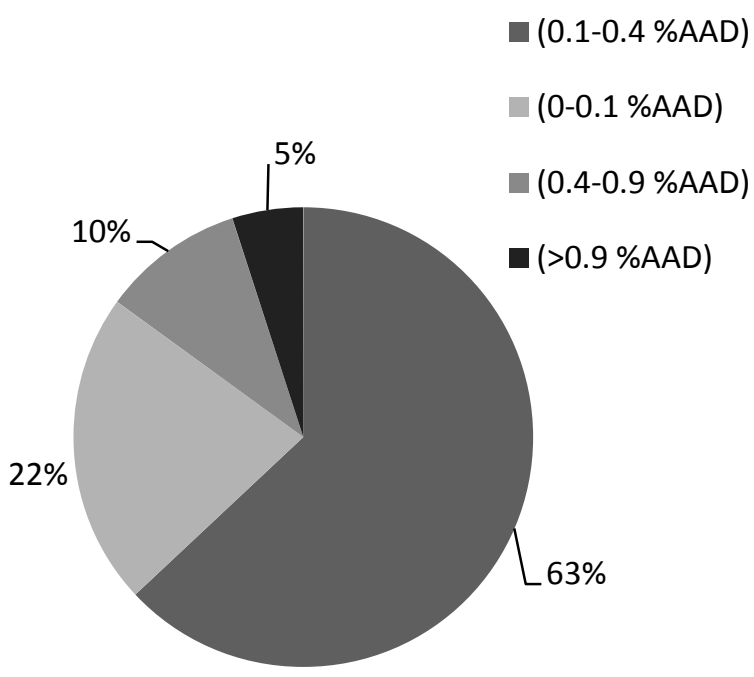

Figure 4: The error distribution of temperature predictions for ternary systems using the NRTL-QSPR model 


\subsection{UNIQUAC models}

The results for the case studies involving the UNIQUAC model are presented in Tables 5-7. The overall statistics are shown for both the UNIQUAC-Regressed and UNIQUAC-QSPR models. The UNIQUAC-Regressed model (Case V) applied to the binary systems resulted in overall \%AADs of $1.7,0.1,3.0$, and 3.7 for $P, T, y_{1}$, and $K$-values, respectively. For the ternary systems with the same model parameters the prediction \%AADs are 2.9, 0.2, 8.5, and 8.0 for $P, T, y_{1}$, and $K$-values, respectively. Similarly, in this case, the ternary prediction errors are within two times of those observed for the binary systems.

For the generalized binary system predictions of the UNIQUAC-QSPR model (Case VI), the \%AADs are 3.0, 0.3, 4.7, and 6.0 for $P, T, y_{1}$, and $K$-values predictions, respectively. Applying the same parameters to ternary mixtures produced \%AADs of $3.5,0.3,8.2$, and $7.9 \%$ AADs for $P, T, y_{1}$, and $K$-values predictions, respectively. As such, the generalized results for both the binary and ternary systems show overall errors that are less than two times the errors produced the regressed parameters in Case $\mathrm{V}$.

Table 7 presents the results for Case VII, which involved 29 ternary systems with at least one missing constituent binary. The UNIQUAC-QSPR model was utilized to predict the parameters for all the constituent binaries. For this case, the overall \%AADs are 8.4, 0.7, 12.9 and 12.5 \%AADs for $P, T, y_{1}$, and $K$-values predictions, respectively. Further, the resulting property prediction errors are less than 2.5 times the error observed in comparison to the results from Case VI, where all the constituent binary systems were included in the database. This indicates the ability of the UNIQUAC-QSPR model to predict equilibrium phase properties of multicomponent mixtures when some constituent binaries are not represented in the database used for model development; albeit, the NRTL-QSPR model provides better results for this case.

Table 5: Case $\mathrm{V}$ results for binary and ternary system phase behavior predictions

\begin{tabular}{|c|c|c|c|c|c|c|c|}
\hline Model & System & Property & No. of sys. & No. of pts. & RMSE & Bias & $\% A A D$ \\
\hline \multirow{4}{*}{$\begin{array}{c}\text { IG / } \\
\text { UNIQUAC }\end{array}$} & \multirow{4}{*}{ Binary } & $\mathrm{P}$ (bar) & \multirow{2}{*}{75} & \multirow{2}{*}{2992} & 0.00 & -0.002 & 1.7 \\
\hline & & $\mathrm{T}(\mathrm{K})$ & & & 2.50 & 0.144 & 0.1 \\
\hline & & $\mathrm{y}_{1}$ & \multirow{2}{*}{63} & \multirow{2}{*}{1818} & 0.00 & 0.001 & 3.0 \\
\hline & & K-values & & & 0.60 & -0.014 & 3.7 \\
\hline \multirow{4}{*}{$\begin{array}{c}\text { IG / } \\
\text { UNIQUAC }\end{array}$} & \multirow{4}{*}{ Ternary } & $P$ (bar) & \multirow{2}{*}{57} & \multirow{2}{*}{2212} & 0.00 & 0.00 & 2.9 \\
\hline & & $\mathrm{T}(\mathrm{K})$ & & & 1.50 & 0.10 & 0.2 \\
\hline & & $\mathrm{y}_{1}$ & \multirow{2}{*}{52} & \multirow{2}{*}{1890} & 0.00 & 0.00 & 8.5 \\
\hline & & K-values & & & 0.40 & 0.00 & 8.0 \\
\hline
\end{tabular}


Table 6: Case VI results for binary and ternary system phase behavior predictions

\begin{tabular}{|c|c|c|c|c|c|c|c|c|}
\hline Model & System & Property & No. of sys. & No. of pts. & RMSE & Bias & $\% A A D$ & $\begin{array}{c}\text { Gen/Reg } \\
\text { Ratio } \\
\end{array}$ \\
\hline \multirow{4}{*}{$\begin{array}{c}\text { IG / } \\
\text { UNIQUAC- } \\
\text { QSPR }\end{array}$} & \multirow{4}{*}{ Binary } & $\mathrm{P}$ (bar) & \multirow{2}{*}{75} & \multirow{2}{*}{3209} & 0.00 & -0.006 & 3.0 & 1.7 \\
\hline & & $\mathrm{T}(\mathrm{K})$ & & & 2.70 & 0.432 & 0.3 & 3.0 \\
\hline & & $\mathrm{y}_{1}$ & \multirow{2}{*}{63} & \multirow{2}{*}{1817} & 0.00 & -0.002 & 4.7 & 1.6 \\
\hline & & K-values & & & 0.70 & -0.064 & 6.0 & 1.6 \\
\hline \multirow{4}{*}{$\begin{array}{c}\text { IG / } \\
\text { UNIQUAC- } \\
\text { QSPR }\end{array}$} & \multirow{4}{*}{ Ternary } & $\mathrm{P}$ (bar) & \multirow{2}{*}{57} & \multirow{2}{*}{2212} & 0.00 & -0.012 & 3.5 & 1.2 \\
\hline & & $\mathrm{T}(\mathrm{K})$ & & & 1.70 & 0.460 & 0.3 & 1.5 \\
\hline & & $\mathrm{y}_{1}$ & \multirow{2}{*}{52} & \multirow{2}{*}{1890} & 0.00 & 0.002 & 8.2 & 1.0 \\
\hline & & K-values & & & 0.40 & 0.008 & 7.9 & 1.0 \\
\hline
\end{tabular}

Table 7: Case VII results for binary and ternary system phase behavior predictions

\begin{tabular}{ccccccc}
\hline Model & Property & No. of sys. & No. of pts. & RMSE & Bias & \%AAD \\
\hline IG / & $\mathrm{P}(\mathrm{bar})$ & & \multirow{2}{*}{981} & 0.12 & 0.03 & 8.4 \\
UNIQUAC- & $\mathrm{T}(\mathrm{K})$ & \multirow{2}{*}{29} & & 3.25 & -0.78 & 0.7 \\
QSPR & $\mathrm{y}_{1}$ & & \multirow{2}{*}{966} & 0.07 & 0.01 & 12.9 \\
& K-values & & & 1.53 & 0.00 & 12.5 \\
\hline
\end{tabular}

Figures 5-8 show the error distribution of the overall \%AADs of the pressure and temperature for the binary and ternary predictions using the UNIQUAC-QSPR (Case V) model parameters. The AAD\% values for pressure and temperature predictions for both the binary and ternary systems are less than 3.0 and 0.4 , respectively, for over $50 \%$ of the data considered. 


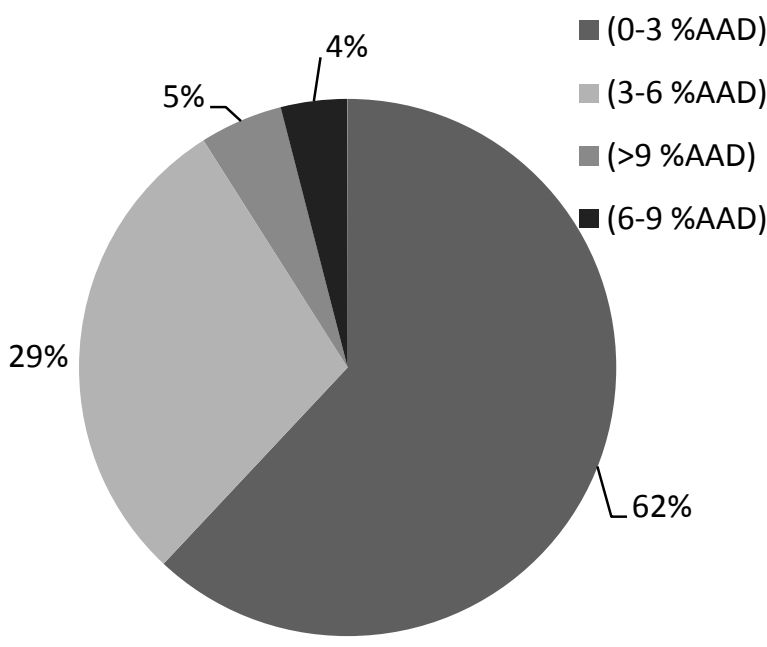

Figure 5: The error distribution of pressure predictions for binary systems using the UNIQUAC-QSPR model

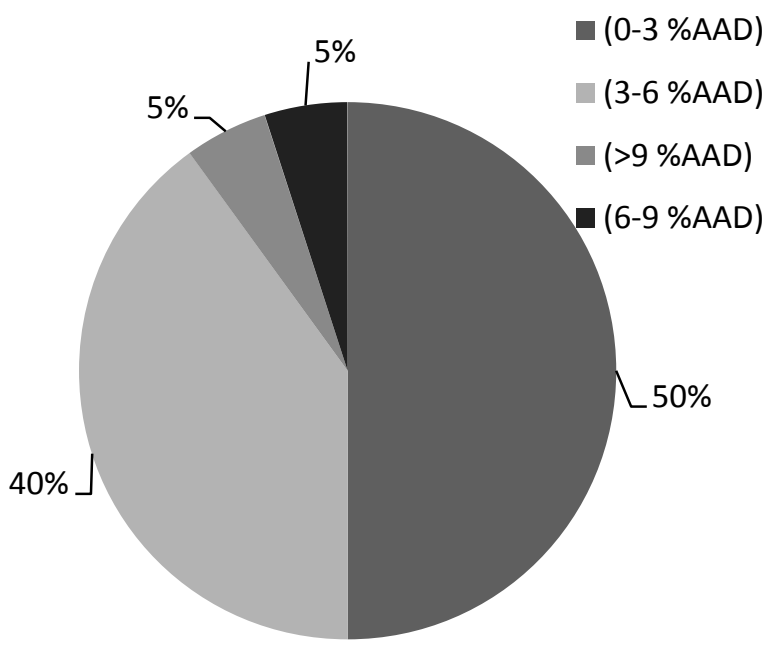

Figure 7: The error distribution of pressure predictions for ternary systems using the UNIQUAC-QSPR model

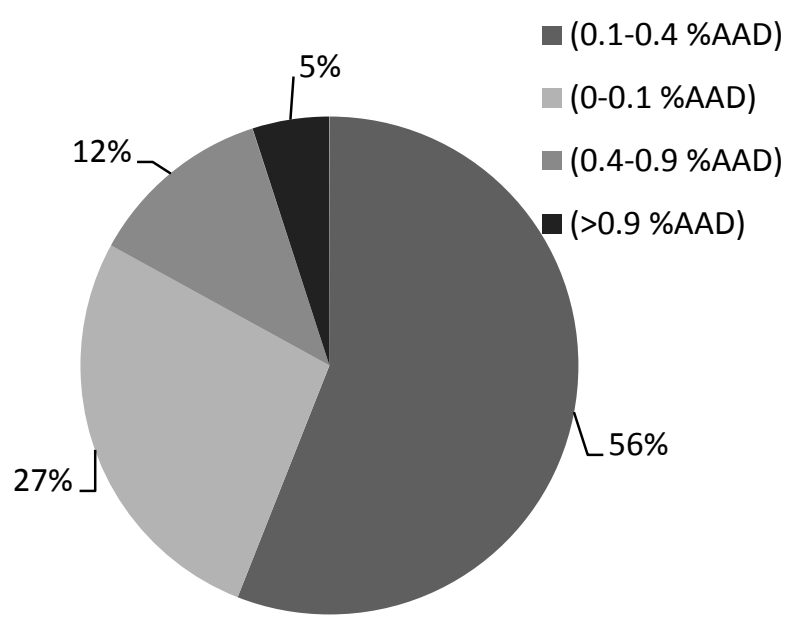

Figure 6: The error distribution of temperature predictions for binary systems using the UNIQUAC-QSPR model

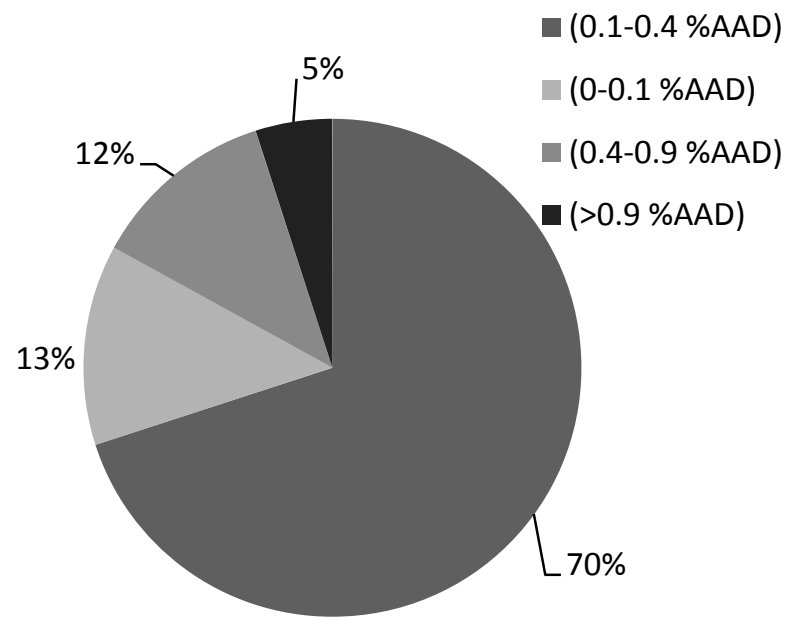

Figure 8: The error distribution of temperature predictions for ternary systems using the UNIQUAC-QSPR model 


\subsection{The $M U N I F A C$ model}

The results for comparing our generalized model with the widely used mUNIFAC model are presented in Tables 8-10. For the 57 ternary systems in which all the constituent binaries are presented, the overall \%AADs of $2.5,0.2,7.8$, and 7.4 were obtained for $P, T, y_{1}$, and $K$-values, respectively. These results are slightly better than those obtained using our generalized NRTL or UNIQUAC models.

The errors are shown in Table 9 for the mUNIFAC model applied to 29 ternary systems that have at least one missing constituent binary. Overall, \%AADs of 20.7, 2.1, 26.6, and 26.7 were obtained for $P, T, y_{1}$, and $K$-values, respectively. This shows more than three times higher error for $P$ and $T$ compared to the NRTL-QSPR model. Similarly, the UNIFAC-6 model yielded more than two times higher error for $P$ and $T$ compared to the UNIQUAC-QSPR model.

Table 8: MUNIFAC prediction results for ternary system phase behavior with all binaries

\begin{tabular}{ccccccc}
\hline Model & Property & No. of sys. & No. of pts. & RMSE & Bias & \%AAD \\
\hline \multirow{3}{*}{ mUNIFAC } & $\mathrm{P}(\mathrm{bar})$ & & \multirow{2}{*}{2212} & 0.04 & 0.00 & 2.5 \\
& $\mathrm{~T}(\mathrm{~K})$ & \multirow{2}{*}{57} & & 1.49 & 0.19 & 0.2 \\
& $\mathrm{y}_{1}$ & & \multirow{2}{*}{1890} & 0.04 & 0.00 & 7.8 \\
& $\mathrm{~K}$-values & & & 0.36 & 0.00 & 8.2 \\
\hline
\end{tabular}

Table 9: mUNIFAC prediction results for ternary system phase behavior with at least one missing binary

\begin{tabular}{ccccccc}
\hline Missing & Property & No. of sys. & No. of pts. & RMSE & Bias & \%AAD \\
\hline \multirow{3}{*}{$\begin{array}{c}\text { At least one } \\
\text { binary }\end{array}$} & $\mathrm{P}(\mathrm{bar})$ & & \multirow{2}{*}{981} & 0.26 & -0.18 & 20.7 \\
& $\mathrm{~T}(\mathrm{~K})$ & \multirow{2}{*}{29} & & 9.71 & 6.92 & 2.1 \\
& $\mathrm{y}_{1}$ & & \multirow{2}{*}{966} & 0.11 & 0.02 & 25.7 \\
& $\mathrm{~K}$-values & & & 2.52 & -0.16 & 26.7 \\
\hline
\end{tabular}


Table 10: Break down of mUNIFAC prediction results for ternary system phase behavior with missing binaries

\begin{tabular}{|c|c|c|c|c|c|c|}
\hline Missing & Property & No. of sys. & No. of pts. & RMSE & Bias & $\% A A D$ \\
\hline \multirow{4}{*}{ One binary } & P (bar) & \multirow{4}{*}{29} & \multirow{2}{*}{981} & 0.19 & -0.12 & 14.2 \\
\hline & $\mathrm{T}(\mathrm{K})$ & & & 7.20 & 4.45 & 1.4 \\
\hline & $\mathrm{y}_{1}$ & & \multirow{2}{*}{966} & 0.10 & 0.02 & 23.4 \\
\hline & K-values & & & 2.38 & -0.13 & 24.3 \\
\hline \multirow{4}{*}{$\begin{array}{c}\text { Two } \\
\text { binaries }\end{array}$} & $\mathrm{P}$ (bar) & \multirow{4}{*}{29} & \multirow{2}{*}{981} & 0.28 & -0.21 & 23.6 \\
\hline & $\mathrm{T}(\mathrm{K})$ & & & 10.82 & 8.03 & 2.4 \\
\hline & $\mathrm{y}_{1}$ & & \multirow{2}{*}{966} & 0.12 & 0.03 & 28.1 \\
\hline & K-values & & & 2.61 & -0.18 & 29.3 \\
\hline \multirow{4}{*}{$\begin{array}{c}\text { Three } \\
\text { binaries }\end{array}$} & P (bar) & & \multirow{2}{*}{981} & 0.36 & -0.27 & 31.8 \\
\hline & $\mathrm{T}(\mathrm{K})$ & 20 & & 13.92 & 11.04 & 3.4 \\
\hline & $\mathrm{y}_{1}$ & 25 & \multirow{2}{*}{966} & 0.11 & 0.03 & 251 \\
\hline & K-values & & & 2.69 & -0.22 & 26.1 \\
\hline
\end{tabular}

As shown in Table 10, for 29 ternary systems with one missing binary constituent, the mUNIFAC model errors are 14.1, 1.4, 23.4, and 24.3 \%AADs for $P, T, y_{1}$, and $K$-values, respectively. For the same set of ternary systems, when two constituent binaries are missing, the errors are 23.5, 2.4, 28.1, and 29.3\%AADs for $P, T, y_{1}$, and $K$-values, respectively. For a case that all three constituent binaries are missing, the mUNIFAC errors are 31.8, 3.3, 25.1, and $26.1 \% A A D s$ for $P, T, y_{1}$, and $K$-values, respectively.

The ultimate goal of any generalized model is to provide reliable a priori predictions for diverse systems, including those for which no experimental data are available. The outcome of this work demonstrates the usefulness of the QSPR binary parameter generalizations for the NRTL and UNIQUAC models for the multicomponent mixtures considered. While additional validation involving a much larger database involving a significantly wider range of structural variations is required, the results obtained indicate the current model generalizations are effective and offer a good complement to existing predictive models, such as mUNIFAC. More importantly, this approach provides accurate predictions for systems lacking group-interaction parameters within mUNIFAC. This evidenced by the results obtained, which show the mUNIFAC model yielding more than two to three times higher error for $P$ and $T$ predictions in comparison with the NRTLQSPR and UNIQUAC-QSPR models when interaction parameters are missing for constituent binaries. 


\section{Summary}

The primary objective of the current study was to investigate representation and prediction of the vapor-liquid equilibrium properties of multicomponent systems using regressed and generalized interaction parameters of constituent binaries. Specifically, we examined the efficacy of four models (NRTL-Regressed, UNIQUAC-Regressed, NRTL-QSPR, and UNIQUACQSPR) in predicting equilibrium properties of ternary systems. In doing so, the regressed models are used as a benchmark for evaluating the efficacy of the generalized models. In addition, the QSPR generalized models were compared with the widely used mUNIFAC model. Following are the conclusions drawn from this study:

- The QSPR parameter generalizations for the NRTL and UNIQUAC models provided useful phase behavior property predictions for both binary and ternary systems.

- The generalized-QSPR models produced VLE predictions within twice the errors obtained by parameter regressions.

- For ternary systems with all binary interactions accounted for, the group-contribution method mUNIFAC produced slightly better results the QSPR generalizations. However, for ternary systems with missing binary interactions, the QSPR models are more accurate than the MUNIFAC model.

- Although additional evaluation using a comprehensive database is required, the results presented demonstrate the efficacy of the NRTL and QSPR binary parameter generalizations for VLE multicomponent property predictions.

\section{Acknowledgement}

This material is based on work partially supported by the U.S. Department of Energy, DOE/EPSCOR (Grant DE-SC0004600). 


\section{References}

[1] A. Arce, A. Blanco, J. Martínez-Ageitos, I. Vidal, Optimization of UNIQUAC structural parameters for individual mixtures; application to new experimental liquid-liquid equilibrium data for aqueous solutions of methanol and ethanol with isoamyl acetate, Fluid Phase Equilibria, 93 (1994) 285-295.

[2] M. Shacham, G. St Cholakov, R.P. Stateva, N. Brauner, Quantitative structure-property relationships for prediction of phase equilibrium related properties, Industrial \& Engineering Chemistry Research, 49 (2010) 900-912.

[3] J. Gmehling, J.D. Li, M. Schiller, A modified UNIFAC model .2. Present parameter matrix and results for different thermodynamic properties, Industrial \& Engineering Chemistry Research, 32 (1993) 178193.

[4] J. Gmehling, D. Tiegs, U. Knipp, A comparison of the predictive capability of different group contribution methods, Fluid Phase Equilibria, 54 (1990) 147-165.

[5] A. Klamt, F. Eckert, COSMO-RS: a novel and efficient method for the a priori prediction of thermophysical data of liquids, Fluid Phase Equilibria, 172 (2000) 43-72.

[6] A. Klamt, F. Eckert, COSMO-RS: a novel and efficient method for the a priori prediction of thermophysical data of liquids (vol 172, pg 43, 2000), Fluid Phase Equilibria, 205 (2003) 357-357.

[7] A. Klamt, V. Jonas, T. Burger, J.C.W. Lohrenz, Refinement and parametrization of COSMO-RS, Journal of Physical Chemistry A, 102 (1998) 5074-5085.

[8] Z.G. Lei, B.H. Chen, C.Y. Li, H. Liu, Predictive molecular thermodynamic models for liquid solvents, solid salts, polymers, and ionic liquids, Chemical Reviews, 108 (2008) 1419-1455.

[9] D. Ravindranath, B.J. Neely, R.L. Robinson, K.A.M. Gasem, QSPR generalization of activity coefficient models for predicting vapor-liquid equilibrium behavior, Fluid Phase Equilibria, 257 (2007) 53-62.

[10] L. Lepori, E. Matteoli, Excess Gibbs energies of the ternary system ehtanol + tetrahydrofuran + cyclohexane at 298.15K, Fluid Phase Equilibria, 134 (1997) 113-131.

[11] R. Reich, M. Cartes, J. Wisniak, H. Segura, Phase equilibria in the ternary system hexane+ethyl 1,1dimethylethyl ether+heptane, Fluid Phase Equilibria, 154 (1999) 99-108.

[12] H. Renon, J.M. Prausnitz, Local compositions in thermodynamic excess functions for liquid mixtures, AIChE, 14 (1968) 135.

[13] P.K. Talley, J. Sangster, C.W. Bale, A.D. Pelton, Prediction of vapor-liquid and liquid-liquid equilibria and thermodynamic properties of multicomponent organic systems from optimized binary data using the Kohler method, Fluid Phase Equilibria, 85 (1993) 101-128.

[14] S. Gebreyohannes, K. Yerramsetty, B.J. Neely, K.A.M. Gasem, Improved QSPR generalized interaction parameters for the nonrandom two-liquid activity coefficient model, Fluid Phase Equilibria, 339 (2013) 20-30.

[15] A. Jakob, H. Grensemann, J. Lohmann, J. Gmehling, Further development of modified UNIFAC (Dortmund): Revision and extension 5, Ind Eng Chem Res, 45 (2006) 7924-7933.

[16] M.S. Walas, Phase equilibrium in chemical engineering, Butterworth Publishers, Stonehem, MA, 1985.

[17] J. Gmehling, U. Onken, W. Arlt, Vapor-liquid equilibrium data collection, chemistry data series, 1977-2001.

[18] D.S. Abrams, J.M. Prausnitz, Statistical thermodynamics of liquid-mixtures-new expression for excess Gibbs energy of partly or completely miscible systems, AIChE, 21 (1975) 116-128.

[19] H. Renon, J.M. Prausnitz, Derivation of 3-parameter Wilson equation for excess Gibbs energy of liquid mixtures, AICHE, 15 (1969) 785.

[20] The OSU VLE Database, in: K.A.M. Gasem (Ed.), Oklahoma State University, 2005.

[21] Dippr project 801, physical and thermodynamic properties of pure chemicals, in, Design Institute for Physical Property Research/AIChE, 2011. 